ISSN 1991-8631

Original Paper

http://indexmedicus.afro.who.int

\title{
Réponse à l'inoculation mycorhizienne de quatre variétés de niébé [Vigna unguiculata (L.) Walp.] cultivées au Burkina Faso et au Sénégal
}

\author{
Hadou HARO ${ }^{1}$, Kadidia B. SANON ${ }^{1 *}$, Ibou DIOP ${ }^{2}$, Aboubacry KANE ${ }^{2}$, \\ Mahamadi DIANDA ${ }^{1}$, Pascal HOUNGNANDAN ${ }^{3}$, Marc NEYRA ${ }^{4}$, Alfred TRAORE ${ }^{5}$ \\ ${ }^{1}$ Laboratoire de Microbiologie, INERA/DPF BP 7047 Ouagadougou 03, BURKINA FASO. \\ ${ }^{2}$ Laboratoire Commun de Microbiologie, Centre IRD-Bel-air, BP 1386 Dakar, Sénégal. \\ ${ }^{3}$ Laboratoire d'écologie Microbienne, Faculté des Sciences Agronomiques (FSA), Université d'Abomey- \\ Calavi (UAC), 01 BP 526 Recette Principale Cotonou, Benin. \\ ${ }^{4}$ IRSTEA Lyon, Equipe EMHA, Ecologie Microbienne des Hydrosystèmes Anthropisés UR MALY/MAEP, \\ Milieux Aquatiques, Ecologie et Pollutions, 3 bis quai Chauveau, CP 220, F-69336 Lyon cedex 09. \\ ${ }^{5}$ Université de Ouagadougou, UFR Science de la Vie et de la Terre, Ecole Doctorale Régionale de \\ Biotechnologies (RA-BIOTECH) 03 BP 7031 Oagadougou 03, BURKINA FASO. \\ *Auteur correspondant; E-mail : sbkady@gmail.com
}

\section{RESUME}

La culture du Niébé [Vigna unguiculata (L.) Walp.] en Afrique de l'Ouest est marquée par l'instabilité de ses rendements et sa faible production liée à la pauvreté des sols, à la faiblesse de la pluviométrie et aux contraintes parasitaires. Dans cette étude, nous nous sommes intéressés à la réponse à l'inoculation mycorhizienne de quatre variétés de niébé dans une perspective de sélection de souches efficaces de champignons endomycorhiziens pour l'amélioration de sa productivité au champ. Quatre variétés de niébé dont deux du Burkina Faso [Gorom Local (GO) et KVX 396-4-5-2D (KVX)] et deux du Sénégal [Mélakh (Mel) et Yacine (Yac)] ont été inoculées en pépinière avec trois (3) inoculums mixtes du Burkina Faso [Yakouta (Ya), Talé Mossi (Ta) et Soumousso (Sou)] et trois inoculums monospécifiques de la collection du Laboratoire Commun de Microbiologie de Dakar (LCM) [Glomus mossea (Gm), Glomus fasciculatum (Gf) et Glomus intraradices (Gi)]. Les effets des différents inoculums sur la croissance des plants ont été évalués par la mesure de la hauteur des plants, des biomasses aérienne et racinaire et de la fréquence et intensité de mycorhization à la floraison et à la fructification. Les résultats obtenus montrent que l'inoculation n'a pas eu d'effets significatifs sur les différents paramètres mesurés pour les variétés du Sénégal malgré des taux de mycorhization de $6 \%$ à $92 \%$ à la floraison et de $44 \%$ à $100 \%$ à la fructification. Pour les variétés du Burkina Faso, à la floraison, la croissance en hauteur et la production de biomasse aérienne de la variété KVX ont été améliorées par toutes les souches, exceptée Gm. Pour la variété GO, les inoculums mixtes indigènes Ya et Ta ont significativement amélioré la croissance en hauteur des plants. En général, les fréquences et les intensités de mycorhization sont élevées pour les souches monospécifiques, mais ceci ne se traduit pas par un effet significatif sur la croissance des plants inoculés. L'inoculum mixte indigène Ya apparaît plus performant pour la croissance en hauteur et la production de biomasse de la variété la plus sensible à l'inoculation (KVX). Cette étude a montré que l'inoculation endomycorhizienne a un effet bénéfique sur la croissance du Niébé, particulièrement la variété KVX ; et que l'utilisation de souches indigènes conviendrait mieux que les souches monospécifiques. Il s'avère donc intéressant de poursuivre la sélection de souches performantes pour la productivité du Niébé sur les souches indigènes.

Mots clés: champignons mycorhiziens, croissance du Niébé, pépinière, Afrique de l'Ouest. 


\section{INTRODUCTION}

Le niébé [Vigna unguiculata (L.) Walp.] est une légumineuse cultivée sur le continent Africain ainsi que dans certaines parties d'Asie du Sud et de l'Amérique latine. Bien que natif de l'Afrique de l'Ouest, cette légumineuse est devenue une partie de l'alimentation d'environ 110 millions de personnes (Ogbuinya, 1997). Avec une teneur en protéines d'environ $25 \%$, cette légumineuse est une source de protéine moins chère pour les pauvres. En Afrique de l'Ouest, où plus de $70 \%$ de la production mondiale totale est cultivée, le niébé est devenu une partie intégrante des systèmes agricoles. Il est adapté à un large éventail de sols et de conditions d'humidité. Toutefois, les rendements moyens ne peuvent être atteints que si sa culture est accompagnée par l'usage de fortes doses d'insecticides ou des mesures de gestion spécifiques, telles que la culture intercalaire ou la polyculture (Ogbuinya, 1997). De plus, sa culture est marquée par l'instabilité de ses rendements et la faiblesse de sa production liée à la pauvreté des sols (Bado, 2002), à la baisse de la pluviométrie et aux contraintes parasitaires (Singh et al., 1997). L'apport d'engrais et de pesticide pourrait constituer une solution à cette faible production. Cependant, leur utilisation a montré ses limites : pollution et perte de biodiversité dans les régions d'agriculture intensive, dégradation des systèmes agricoles les plus fragiles et coûts prohibitifs pour les producteurs les plus pauvres (Plenchette et al., 2005). Ainsi, l'agriculture conventionnelle doit s'orienter vers des systèmes de cultures plus durables à faibles intrants.Parmi les systèmes de cultures les plus durables et les pratiques permettant d'espérer une amélioration de cette production, se trouve la technique d'inoculation des plantes avec des microorganismes symbiotiques présents naturellement dans le sol, notamment les champignons mycorhiziens. En effet, la symbiose mycorhizienne, une relation impliquant un échange bidirectionnel des ressources entre les plantes hôtes et certains champignons du sol, est une des associations biologiques les plus communes et largement étudiées entre des plantes et des microorganismes. Dans cette association à bénéfice réciproque, les champignons sont susceptibles de conférer à leurs hôtes, une meilleure croissance grâce à l'amélioration de la nutrition hydrominérale, en particulier la nutrition phosphatée et la réduction sensible de la pression parasitaire ; par conséquent un meilleur rendement (Egli et Brunner, 2002). En retour, ces champignons bénéficient des ressources énergétiques et carbonées de la plante nécessaires à leur croissance et leur développement (Marx, 1969; Smith et Read, 1997). L'amélioration de l'absorption de phosphore, de la nutrition et de la croissance des plantes cultivées par les champignons mycorhiziens à arbuscules (CMA) a été beaucoup documentée (Mosse, 1973; Rahman et Parsons, 1997; Rahman et al., 2006 ; Diouf et al., 2009; Megueni et al., 2011). Ainsi, les CMA apparaissent comme un composant indispensable d'une agriculture durable, d'où la nécessité d'en accélérer l'intégration dans les systèmes agricoles.

Le niébé a l'avantage de vivre en symbiose avec les champignons mycorhiziens à arbuscules et des bactéries du sol fixatrices d'azote atmosphérique. Dans cette étude, nous nous sommes intéressés à la réponse à l'inoculation mycorhizienne de quatre (4) variétés de niébé cultivées au Burkina Faso et au Sénégal dans une perspective de sélectionner des souches efficaces pour l'amélioration de la productivité du Niébé au champ.

\section{MATERIEL ET METHODES Matériel végétal}

Pour évaluer la sensibilité et l'efficacité de la mycorhization du niébé, deux variétés les plus cultivées au Burkina Faso (Gorom local et KVX 396-4-5-2D) et deux variétés les 
plus cultivées au Sénégal (Mélakh et Yacine) ont été utilisées. Les caractéristiques de ces 4 variétés sont présentées dans le Tableau 1 (Ouédraogo et al., 2003).

\section{Matériel fongique}

Trois (3) inoculums fongiques de la collection du Laboratoire Commun de Microbiologie (LCM) du Sénégal (Glomus mossea $(\mathrm{Gm})$, Glomus fasciculatum (Gf) et Glomus intraradices (Gi) et trois (3) inoculums mixtes du Burkina Faso ont été utilisés. Les inoculums mixtes ont été obtenus par multiplication des souches de champignons mycorhiziens à arbuscules indigènes des sols de trois zones climatiques du Burkina Faso [zone sahélienne (Yakouta), zone sahélo-soudanienne (Talé Mossi) et zone soudanienne (Soumousso)].

\section{Production d'inoculum des souches indigènes}

La production d'inoculum a consisté à cultiver sous serre, une plante mycotrophe, le petit mil (Pennisetum glaucum) dans des pots contenant $250 \mathrm{~g}$ de sol (sol de différents champs : Yakouta, Talé Mossi et Soumousso contenant des champignons indigènes) et $1750 \mathrm{~g}$ de sable stérilisé à $120^{\circ} \mathrm{C}$ pendant $1 \mathrm{~h}$. Les propriétés physico-chimiques de ce sable sont: $\mathrm{pH}$ eau $(5,7)$, argile $(3,9 \%)$, limon $(4,8 \%)$, sable $(91,2 \%), \mathrm{C}$ total $(0,12 \%), \mathrm{N}$ total (74 ppm), P total (61 ppm), P Bray (2,6 ppm).

Chaque traitement a été répété 3 fois. Les témoins ont reçu chacun $2000 \mathrm{~g}$ de sable stérile. Ces pots ont été arrosés régulièrement à la capacité au champ et recevaient tous les 15 jours, $100 \mathrm{ml}$ d'une solution de Long Ashton (Hewitt, 1966). L'inoculum est obtenu après 3 mois de culture et est constitué d'un mélange de spores, de fragments de racines mycorhizées et de sable.

\section{Traitement des graines et germination}

Les graines de niébé ont été aseptisées par trempage dans l'éthanol $96^{\circ}$ pendant 3 minutes, rincées abondamment à l'eau distillée stérile puis trempées de nouveau dans une solution d'hypochlorite de calcium $\left(\mathrm{CaCl}_{2} \mathrm{O}_{2}\right.$ à $\left.3,3 \% ; \mathrm{p} / \mathrm{v}\right)$ pendant $3 \mathrm{~min}$ et rincées abondamment à l'eau distillée stérile. Elles ont ensuite été repiquées dans des boites de Pétri contenant de l'eau gélosée $(0,8 \%)$ et mises à germer à $30{ }^{\circ} \mathrm{C}$ dans une étuve. Après 48 heures, les graines germées, sont débarrassées de leurs téguments et repiquées dans les pots de $1 \mathrm{~L}$ inoculés ou non.

\section{Substrat de culture, inoculation et dispositif expérimental}

Le substrat de culture est un sol sableux prélevé à Sangalkam (50 km à l'est de Dakar) et stérilisé $1 \mathrm{H}$ à $120{ }^{\circ} \mathrm{C}$. Les propriétés physico-chimiques de ce substrat sont les suivantes : $\mathrm{pH} \mathrm{H}_{2} \mathrm{O}(6,5)$, argile $(3,6 \%)$, limon $(32,8 \%)$, sable $(58,15 \%), \mathrm{C}$ total $(0,54 \%), \mathrm{N}$ total $(0,06 \%)$, rapport $\mathrm{C} / \mathrm{N}(8,5), \mathrm{P}$ total $(39$ $\mathrm{ppm})$ et P Olsen (4,8 ppm).

L'inoculation a lieu au moment du repiquage des graines pré-germées. Elle a consisté à enfoncer au milieu de chaque pot contenant le substrat de culture, $10 \mathrm{~g}$ d'inoculum à 2 ou $3 \mathrm{~cm}$ de profondeur. Les pots témoins n'ont pas reçu d'inoculum. Les graines pré-germées ont été repiquées à raison de 2 graines par pot, qui sont ramenées à une plantule au bout de deux semaines. Un total de 4 variétés $x 7$ inoculums (dont un témoin) soit 28 traitements répétés chacun 10 fois a été retenu. Le dispositif est de type randomisation simple sous serre. Les plants ont été arrosés régulièrement à l'eau de robinet.

\section{Paramètres mesurés et analyse statistique}

Cinq plants par traitement ont été récoltés à 25 jours après semis (stade de floraison) et les cinq autres à 60 jours après semis (stade de fructification).

Pour estimer les effets des différents inoculums au $25^{\mathrm{e}}$, puis au $60^{\mathrm{e}}$ jour après semis, la hauteur des plants a été mesurée et les biomasses aériennes et racinaires ont été évaluées après séchage à l'étuve à $70^{\circ} \mathrm{C}$ 
pendant 4 jours. Les paramètres de mycorhization ont également été mesurés sur des échantillons de racines fines colorées suivant la méthode décrite par Phillips et Hayman (1970). La fréquence et l'intensité de mycorhization ont été évaluées sous microscope selon la méthode de Trouvelot et al. (1986) au grossissement GX100.

Toutes les données collectées pour les différents paramètres ont été soumises à une analyse de variance (ANOVA) avec le logiciel statistique XLSTAT-Pro 7.5 et les moyennes des variables ont été comparées en utilisant le test de Newman Keuls au seuil de probabilité $\mathrm{p}=5 \%$. Les valeurs pour la fréquence et l'intensité de mycorhization ont été transformées par la formule $\operatorname{arsin} \sqrt{ }$ avant d'être analysées

\section{RESULTATS}

\section{Hauteur des plants à la floraison et à la fructification}

La Figure 1 présente la hauteur des plants à la floraison et à la fructification. La hauteur des plants varie en fonction de la variété de niébé et de l'inoculum utilisé. La variété Mel a une croissance relativement plus importante que celle des autres variétés.

Pour la variété KVX, les analyses statistiques montrent des différences significatives entre les plants inoculés et les témoins et entre les plants inoculés avec Yakouta (Ya) et les autres traitements à la floraison et à la fructification. Les valeurs les plus élevées sont obtenues avec l'inoculum Ya. Pour la variété Gorom local (GO), les analyses statistiques ne montrent pas de différences significatives entre les plants inoculés et les témoins à la fructification, cependant, à la floraison les inoculums Talé mossi (Ta) et Ya induisent une meilleure croissance. Les valeurs les plus élevées ont également été obtenues avec l'inoculum Ya.

Les analyses statistiques ne montrent aucune différence significative entre les plants inoculés et les témoins pour les variétés Mel et Yac.
Il ressort de ces résultats que les variétés du Burkina Faso, en particulier KVX, sont les plus sensibles à l'inoculation pour leur croissance en hauteur, et la variété Yac du Sénégal la moins sensible.

\section{Biomasse}

L'analyse statistique des données sur les biomasses aérienne et racinaire des différents traitements récoltés à différents stades (floraison et fructification) a montré une variabilité entre les variétés (Figures 2 et 3 ). Les différences entre les traitements ont été observées essentiellement pour la variété KVX. En effet, la biomasse aérienne de tous les plants inoculés de cette variété, exceptée avec Glomus mossea (Gm), est significativement plus grande que chez les plants témoins à la floraison. Pour la biomasse souterraine, seuls les plants inoculés avec Glomus intraradices (Gi) et $\mathrm{Ya}$ ont une biomasse statistiquement supérieure à celle des témoins. A la fructification, les plus importantes biomasses aériennes ont été obtenues avec les souches Ya, Ta, Soumousso (Sou) et Glomus fasciculatum (Gf). Exceptée la variété KVX, aucun effet significatif par rapport aux témoins n'a été observé pour la biomasse racinaire des variétés GO, Yac et Mel. Concernant la variété KVX, des biomasses racinaires plus élevées ont été obtenues avec les souches Ya et Gi. On observe une diminution de cette biomasse à la fructification.

Comme pour la croissance en hauteur, les plants inoculés ou non des variétés sénégalaises n'ont pas montré de différences significatives ni à la floraison, ni à la fructification par rapport aux témoins. Il en est de même pour GO.

\section{Paramètres de mycorhization}

Le Tableau 2 présente les résultats de la fréquence et de l'intensité de mycorhization. De manière générale, la fréquence et l'intensité de mycorhization augmentent entre le $25^{\mathrm{e}}$ et le $60^{\mathrm{e}}$ jour après semis. Cette 
augmentation serait due à la forte demande en phosphore des plantes à la fructification. Les analyses statistiques montrent une différence entre tous les traitements inoculés et les témoins. Aucun témoin n'a été mycorhizé suggérant que les différences observées pour les paramètres mesurés sont essentiellement dues à l'effet des souches inoculées. A la première récolte ( 25 jours), la variété $\mathrm{GO}$ est fortement mycorhizée avec les souches monospécifiques ( $85 \%$ à $86 \%$ ) par rapport aux souches mixtes (45\% à $61 \%)$, avec des différences significatives entre ces traitements. L'intensité de mycorhization apparaît corrélée avec la fréquence. Pour les autres variétés, il existe une variabilité entre les souches mixtes et monospécifiques. En effet, la variété KVX présente les plus fortes fréquences avec les souches Ta $(97 \%)$ et Gi
$(89 \%)$; avec également les plus fortes intensités de mycorhization, $77,35 \%$ et $47,71 \%$ respectivement. $\mathrm{La}$ variété Mel inoculée avec Ta présente la meilleure fréquence et intensité de mycorhization $(92 \%$ et $48,76 \%$ ) par rapport aux autres souches. Quant à Yac, les meilleures fréquences et intensités sont obtenues avec Ya $(88 \%$ et $55,12 \%)$ et $\mathrm{Gi}(84 \%$ et $55,59 \%)$. A la fructification (60 jours), les fréquences et surtout les intensités de mycorhization sont en général plus élevées. Les souches monospécifiques présentent les meilleures fréquences et intensités de mycorhization pour toutes les variétés. La fréquence et l'intensité de mycorhization sont très élevées et atteignent parfois $100 \%$ comme taux de mycorhization pour $\mathrm{GO}+\mathrm{Gf}, \mathrm{GO}+\mathrm{Gm}$ et Yac+Gm à la fructification (Tableau 2).

Tableau 1: Caractéristiques des quatre variétés de niébé inoculées en pépinière.

\begin{tabular}{|c|c|c|c|c|}
\hline Variétés & KVX 396-4-5-2D & Gorom local & Mélakh & Yacine \\
\hline Origine & Burkina Faso & Burkina Faso & Sénégal & Sénégal \\
\hline Cycle (jours) & 70 & 70 & 64 & $64-70$ \\
\hline Couleur & Blanche & Brune & Blanche & Rouge \\
\hline Qualité & Bonne & $\begin{array}{l}\text { Légèrement } \\
\text { sucrée }\end{array}$ & $\begin{array}{c}\text { Sec ou haricot } \\
\text { vert }\end{array}$ & $\mathrm{Sec}$ \\
\hline $\begin{array}{l}\text { Sensibilité aux } \\
\text { insectes }\end{array}$ & $\begin{array}{c}\text { Trips }+ \\
\text { Pucerons + } \\
\text { Punaises + }\end{array}$ & $\begin{array}{c}\text { Trips }+++ \\
\text { Pucerons }+++ \\
\text { Punaises }+++\end{array}$ & $\begin{array}{c}\text { Trips - } \\
\text { Puceron - }\end{array}$ & $\begin{array}{c}\text { Aphids - Bruchid + } \\
\text { Trips + Amsacta+ } \\
\text { Puceron- }\end{array}$ \\
\hline Maladies & $\begin{array}{c}\text { Chancre bactérien - } \\
\text { Taches brunes - } \\
\text { Virose - }\end{array}$ & $\begin{array}{c}\text { Chancre } \\
\text { bactérien - } \\
\text { Taches brunes - } \\
\text { Virose ++ }\end{array}$ & $\begin{array}{c}\text { Chancre } \\
\text { bactérien - }\end{array}$ & Chancre bactérien \\
\hline Parasitisme & Striga - & Striga - & & Striga + \\
\hline $\begin{array}{l}\text { Rendement } \\
\text { grain potentiel }\end{array}$ & $1500 \mathrm{~kg} / \mathrm{ha}$ & $1500 \mathrm{~kg} / \mathrm{ha}$ & $1000 \mathrm{~kg} / \mathrm{ha}$ & \\
\hline $\begin{array}{l}\text { Aire de culture } \\
(\mathrm{mm} / \mathrm{an})\end{array}$ & $400 \leq \mathrm{P} \leq 800$ & $400 \leq \mathrm{P} \leq 800$ & & \\
\hline Sécheresse & - & - & - & - \\
\hline
\end{tabular}


Tableau 2: Fréquence et intensité de mycorhization à 25 jours et 60 jours après semis des variétés de niébé GO, KVX, Mel et Yac inoculées ou non avec les souches de champignons mycorhiziens Gf, Gi, Gm, Sou, Ta et Ya.

\begin{tabular}{|c|c|c|c|c|c|}
\hline \multirow[t]{2}{*}{ Variétés } & \multirow[t]{2}{*}{ Inoculum } & \multicolumn{2}{|c|}{ Floraison (25 jours) } & \multicolumn{2}{|c|}{ Fructification (60 jours) } \\
\hline & & Fréquence (\%) & Intensité (\%) & Fréquence (\%) & Intensité (\%) \\
\hline \multirow{7}{*}{ GO } & Gf & $86 \pm 4,0^{\mathrm{a}}$ & $54,73 \pm 5,14^{\mathrm{a}}$ & $100^{\mathrm{a}}$ & $77,16 \pm 3,09^{\mathrm{a}}$ \\
\hline & $\mathrm{Gi}$ & $86 \pm 4,52^{\mathrm{a}}$ & $40,33 \pm 4,68^{\mathrm{b}}$ & $80 \pm 5,77^{\mathrm{bc}}$ & $32,1 \pm 5,19^{b}$ \\
\hline & $\mathrm{Gm}$ & $85 \pm 4,01^{\mathrm{a}}$ & $59.6 \pm 5,95^{\mathrm{a}}$ & $100^{\mathrm{a}}$ & $77,87 \pm 3,22^{\mathrm{a}}$ \\
\hline & Sou & $53 \pm 8,83^{b}$ & $9,63 \pm 2,0^{\mathrm{cd}}$ & $71,67 \pm 6,54^{\mathrm{c}}$ & $11,23 \pm 3,04^{\mathrm{c}}$ \\
\hline & $\mathrm{Ta}$ & $61 \pm 7,22^{\mathrm{b}}$ & $19,4 \pm 2,33^{\mathrm{c}}$ & $67,14 \pm 7,47^{\mathrm{c}}$ & $32,75 \pm 5,38^{b}$ \\
\hline & TS & $0^{\mathrm{c}}$ & $0^{\mathrm{d}}$ & $0^{\mathrm{d}}$ & $0^{\mathrm{d}}$ \\
\hline & Ya & $45 \pm 4,77^{\mathrm{b}}$ & $9,25 \pm 1,81^{\mathrm{cd}}$ & $87,14 \pm 4,21^{\mathrm{ab}}$ & $33,07 \pm 3,31^{\mathrm{b}}$ \\
\hline \multirow{7}{*}{ KVX } & Gf & $60 \pm 5,16^{c}$ & $36,37 \pm 3,49^{\mathrm{bc}}$ & $94 \pm 2,21^{\mathrm{a}}$ & $84,41 \pm 3,34^{\mathrm{a}}$ \\
\hline & $\mathrm{Gi}$ & $89 \pm 3,48^{\mathrm{ab}}$ & $47,71 \pm 4,15^{b}$ & $97,14 \pm 2,85^{\mathrm{a}}$ & $51,07 \pm 4,49^{b}$ \\
\hline & $\mathrm{Gm}$ & $55 \pm 4,77^{\mathrm{c}}$ & $30,93 \pm 4,47^{\mathrm{c}}$ & $95,56 \pm 1,76^{\mathrm{a}}$ & $82,12 \pm 2,43^{\mathrm{a}}$ \\
\hline & Sou & $54 \pm 5,42^{c}$ & $15,25 \pm 1,92^{\mathrm{d}}$ & $83 \pm 4,48^{b}$ & $45,03 \pm 5,36^{b}$ \\
\hline & $\mathrm{Ta}$ & $97 \pm 1,53^{\mathrm{a}}$ & $77,35 \pm 1,41^{\mathrm{a}}$ & $91,43 \pm 3,4^{\mathrm{a}}$ & $58,01 \pm 5,37^{\mathrm{b}}$ \\
\hline & TS & $0^{\mathrm{d}}$ & $0^{\mathrm{e}}$ & $0^{\mathrm{c}}$ & $0^{c}$ \\
\hline & Ya & $82 \pm 6,29^{b}$ & $45,25 \pm 5,25^{\mathrm{b}}$ & $98,57 \pm 1,43^{\mathrm{a}}$ & $79,51 \pm 3,4^{\mathrm{a}}$ \\
\hline \multirow{7}{*}{ Mel } & Gf & $28 \pm 4,16^{\mathrm{d}}$ & $13,56 \pm 2,5^{b}$ & $76 \pm 4,52^{\mathrm{a}}$ & $46,46 \pm 3,47^{b}$ \\
\hline & $\mathrm{Gi}$ & $73 \pm 3,34^{b}$ & $39,63 \pm 3,87^{\mathrm{a}}$ & $90 \pm 3,33^{\mathrm{a}}$ & $32,94 \pm 4^{c}$ \\
\hline & $\mathrm{Gm}$ & $44 \pm 7,18^{\mathrm{c}}$ & $21,45 \pm 4,23^{b}$ & $77 \pm 5,58^{\mathrm{a}}$ & $56,11 \pm 4,32^{\mathrm{a}}$ \\
\hline & Sou & $61 \pm 5,67^{b}$ & $15,17 \pm 4,14^{b}$ & $44 \pm 5,21^{b}$ & $6,43 \pm 2,36^{\mathrm{d}}$ \\
\hline & $\mathrm{Ta}$ & $92 \pm 2^{\mathrm{a}}$ & $48,76 \pm 5,41^{\mathrm{a}}$ & $76 \pm 4,52^{a}$ & $38,77 \pm 4,45^{\mathrm{bc}}$ \\
\hline & TS & $0^{\mathrm{e}}$ & $0^{\mathrm{c}}$ & $0^{\mathrm{c}}$ & $0^{\mathrm{d}}$ \\
\hline & $\mathrm{Ya}$ & $75 \pm 3,72^{\mathrm{b}}$ & $39,67 \pm 5,05^{\mathrm{a}}$ & $0^{*}$ & $0^{*}$ \\
\hline \multirow{7}{*}{ Yac } & Gf & $53 \pm 7,61^{\mathrm{c}}$ & $36,35 \pm 6,94^{b}$ & $97,8 \pm 1,47^{\mathrm{a}}$ & $86,3 \pm 2,11^{\mathrm{a}}$ \\
\hline & $\mathrm{Gi}$ & $84 \pm 3,06^{\mathrm{a}}$ & $55,59 \pm 4,56^{\mathrm{a}}$ & $97,5 \pm 2,5^{\mathrm{a}}$ & $62,9 \pm 3,77^{\mathrm{b}}$ \\
\hline & $\mathrm{Gm}$ & $69 \pm 3,79^{b}$ & $42,62 \pm 2,61^{\mathrm{ab}}$ & $100^{\mathrm{a}}$ & $90,3 \pm 1,98^{\mathrm{a}}$ \\
\hline & Sou & $6 \pm 2,67^{\mathrm{d}}$ & $1,48 \pm 1,39^{\mathrm{d}}$ & $88 \pm 2,49^{b}$ & $46,8 \pm 3,11^{\mathrm{c}}$ \\
\hline & $\mathrm{Ta}$ & $47 \pm 9,07^{\mathrm{c}}$ & $13,93 \pm 3,12^{\mathrm{c}}$ & $0^{*}$ & $0^{*}$ \\
\hline & TS & $0^{\mathrm{d}}$ & $0^{\mathrm{d}}$ & $0^{\mathrm{c}}$ & $0^{\mathrm{d}}$ \\
\hline & Ya & $88 \pm 3,59^{a}$ & $55,12 \pm 4,24^{\mathrm{a}}$ & $86 \pm 3,71^{b}$ & $42,3 \pm 4,63^{\mathrm{c}}$ \\
\hline
\end{tabular}

Pour chaque colonne et pour la même variété de niébé, les valeurs suivies d'une même lettre ne sont pas différentes significativement selon le test de Newman-Keuls au seuil de 5\%; * : plants morts à 60 jours. 

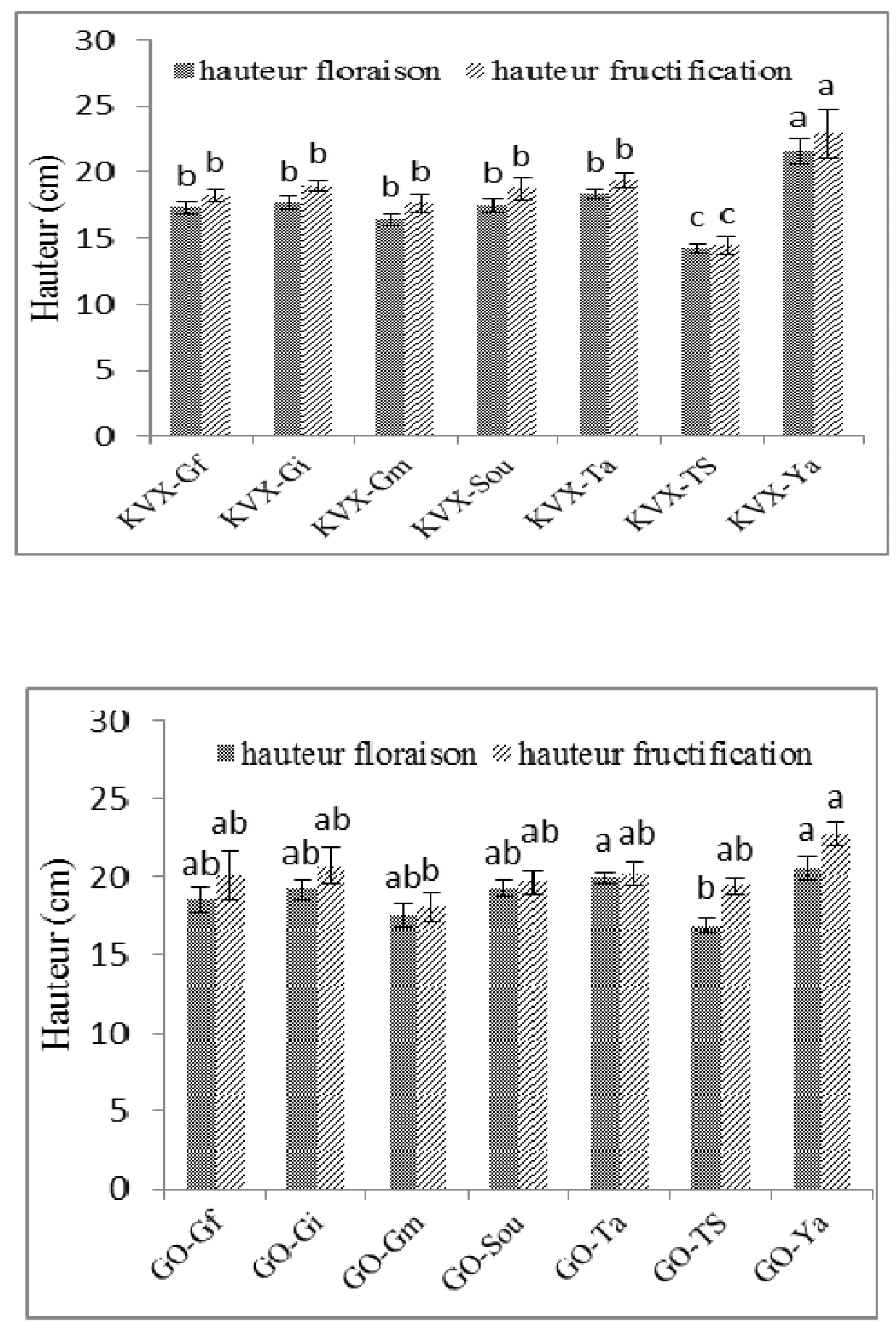

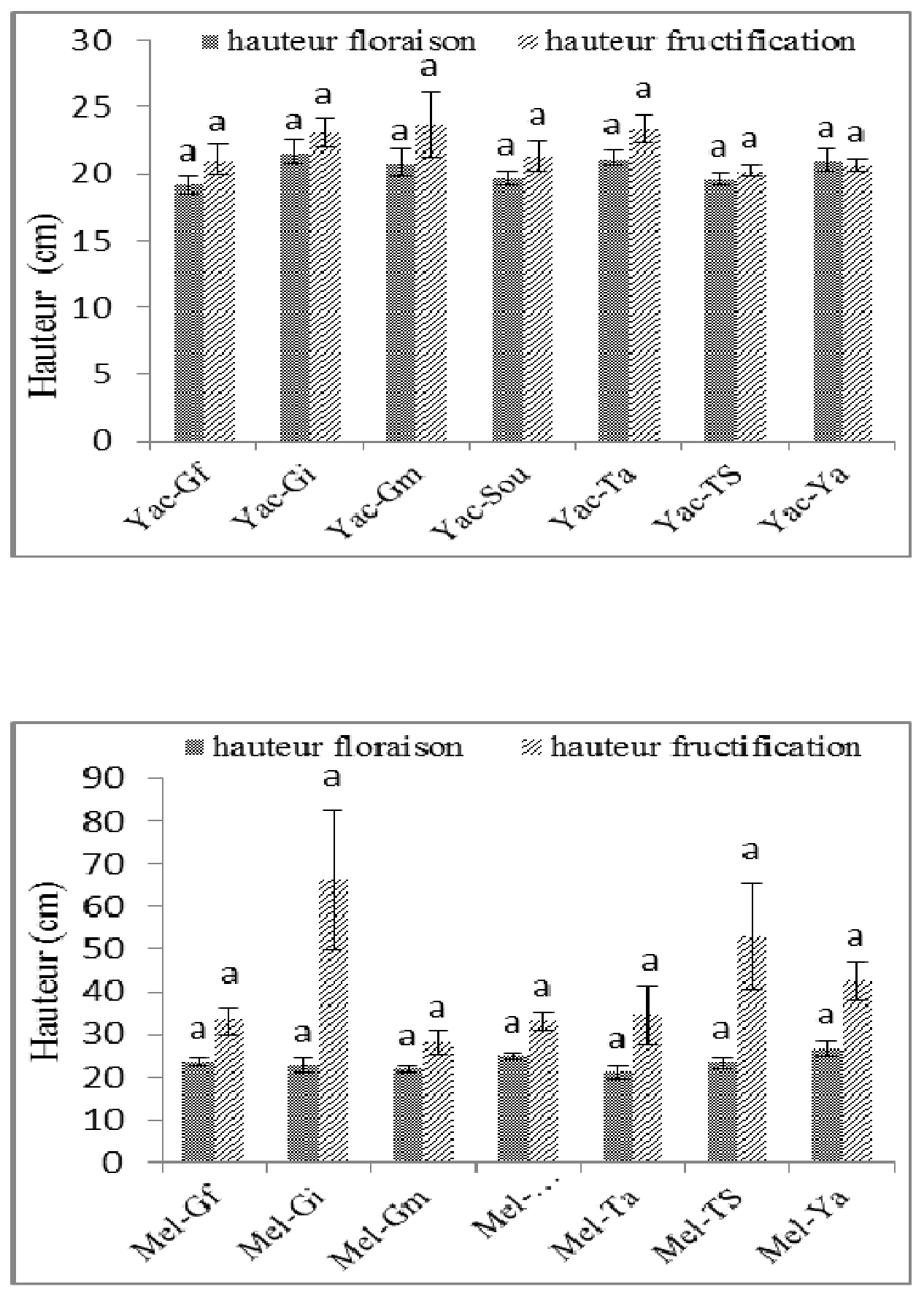

Figure 1: Taille à la floraison et à la fructification des variétés de niébé KVX 396-4-5-2D (KVX), Gorom local (GO), Mélakh (Mel) et Yacine (Yac) inoculées ou non avec les souches de CMA. (Glomus mossea : Gm ; Glomus fasciculatum : Gf ; Glomus intraradices : Gi ; talé mossi : Ta ; yakouta : Ya et soumousso : Sou). TS= témoin stérile. Pour le même paramètre (hauteur à la floraison ou à la fructification) et pour la même variété, les valeurs qui ont en commun une même lettre ne sont pas différentes significativement selon le test de Newman-Keuls au seuil de $5 \%$. 

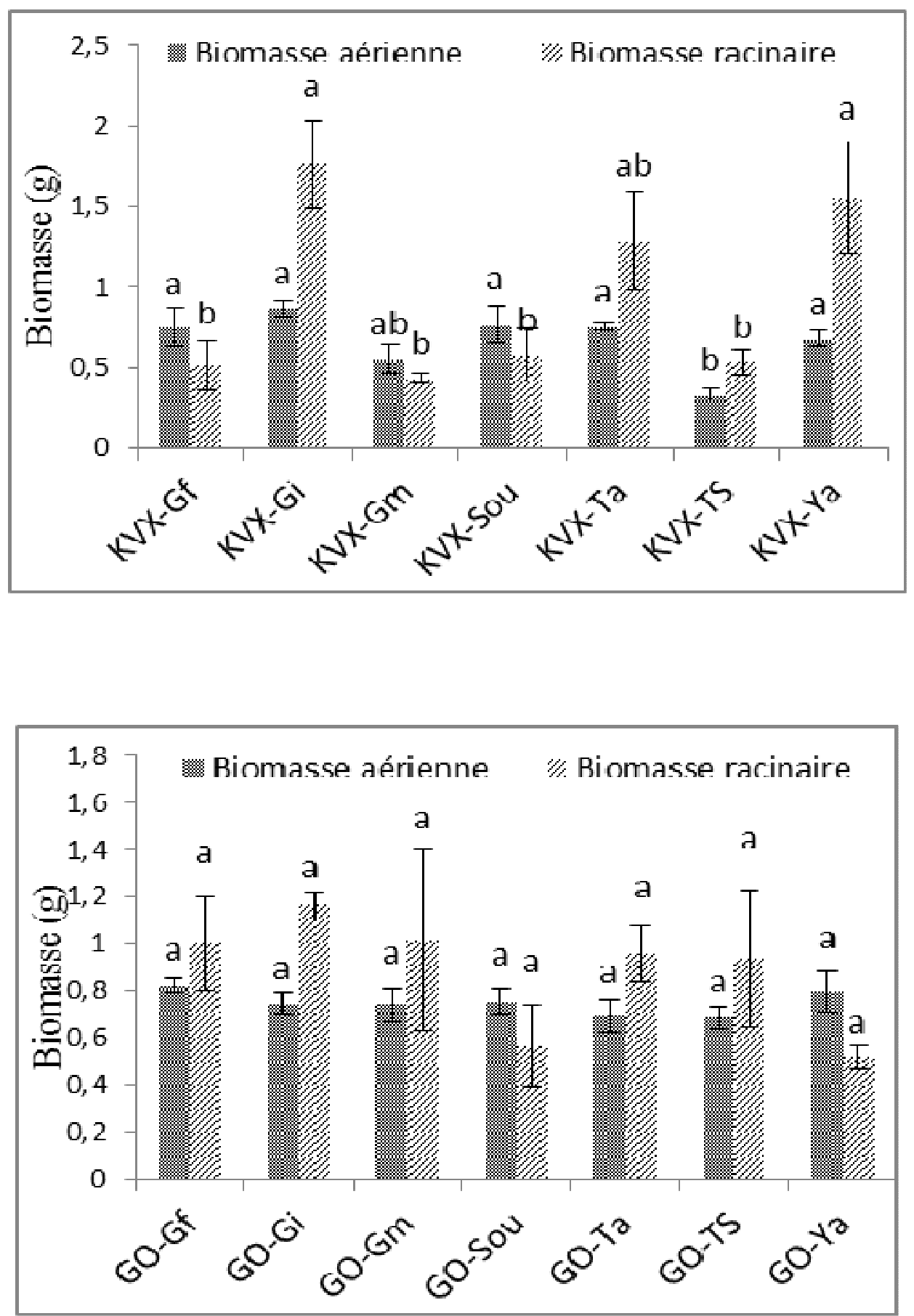

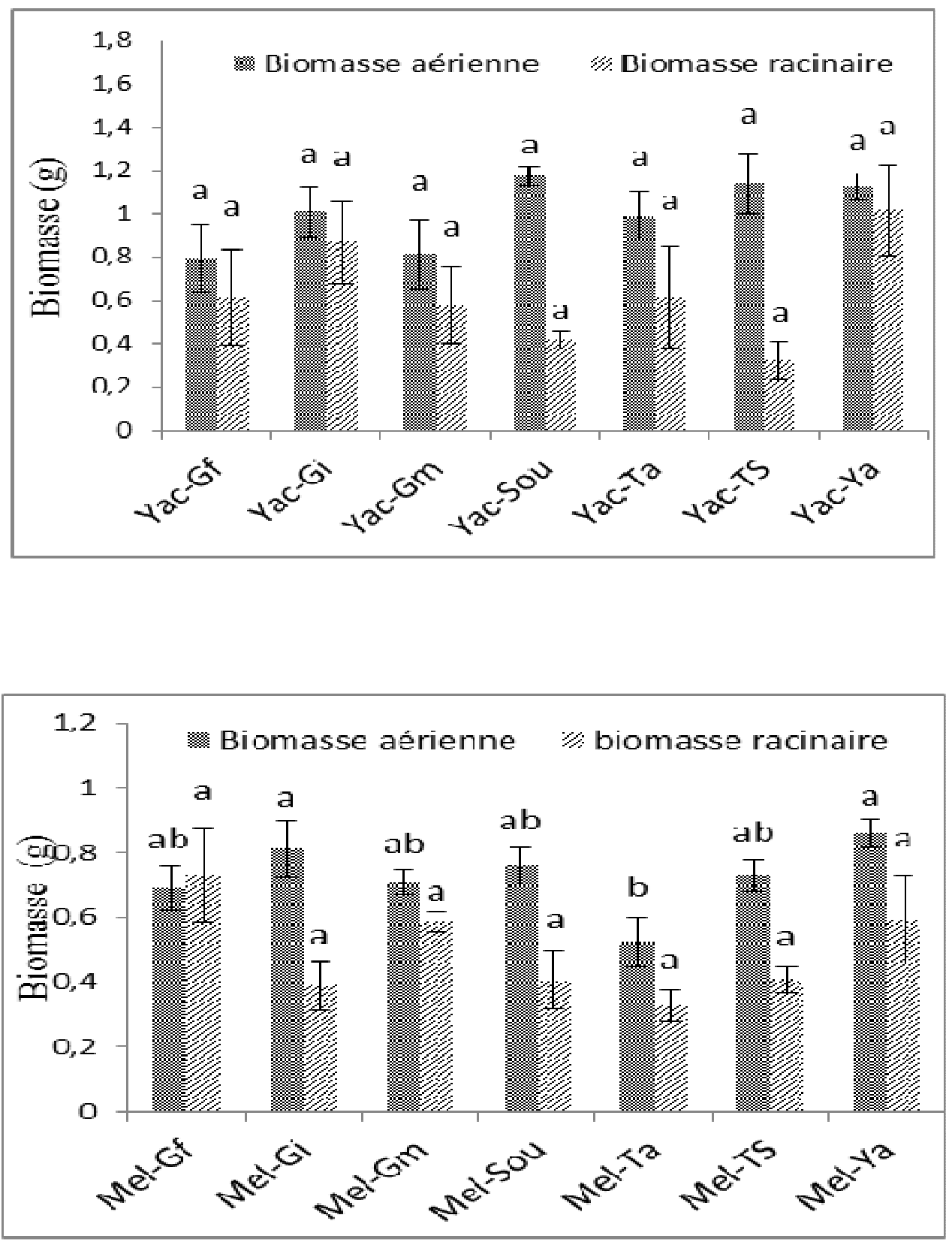

Figure 2: Biomasses aérienne et racinaire après 25 jours de semis des variétés de niébé KVX 3964-5-2D (KVX), Gorom local (GO), Mélakh (Mel) et Yacine (Yac) inoculées ou non avec les souches de CMA. (Glomus mossea : Gm, Glomus fasciculatum : Gf, Glomus intraradices : Gi, talé mossi 1: Ta, yakouta 1: Ya et soumousso1: Sou). TS= témoin stérile.Pour le même paramètre (biomasse aérienne ou racinaire) et pour la même variété, les valeurs qui ont en commun une même lettre ne sont pas différentes significativement selon le test de NewmanKeuls au seuil de $5 \%$. 

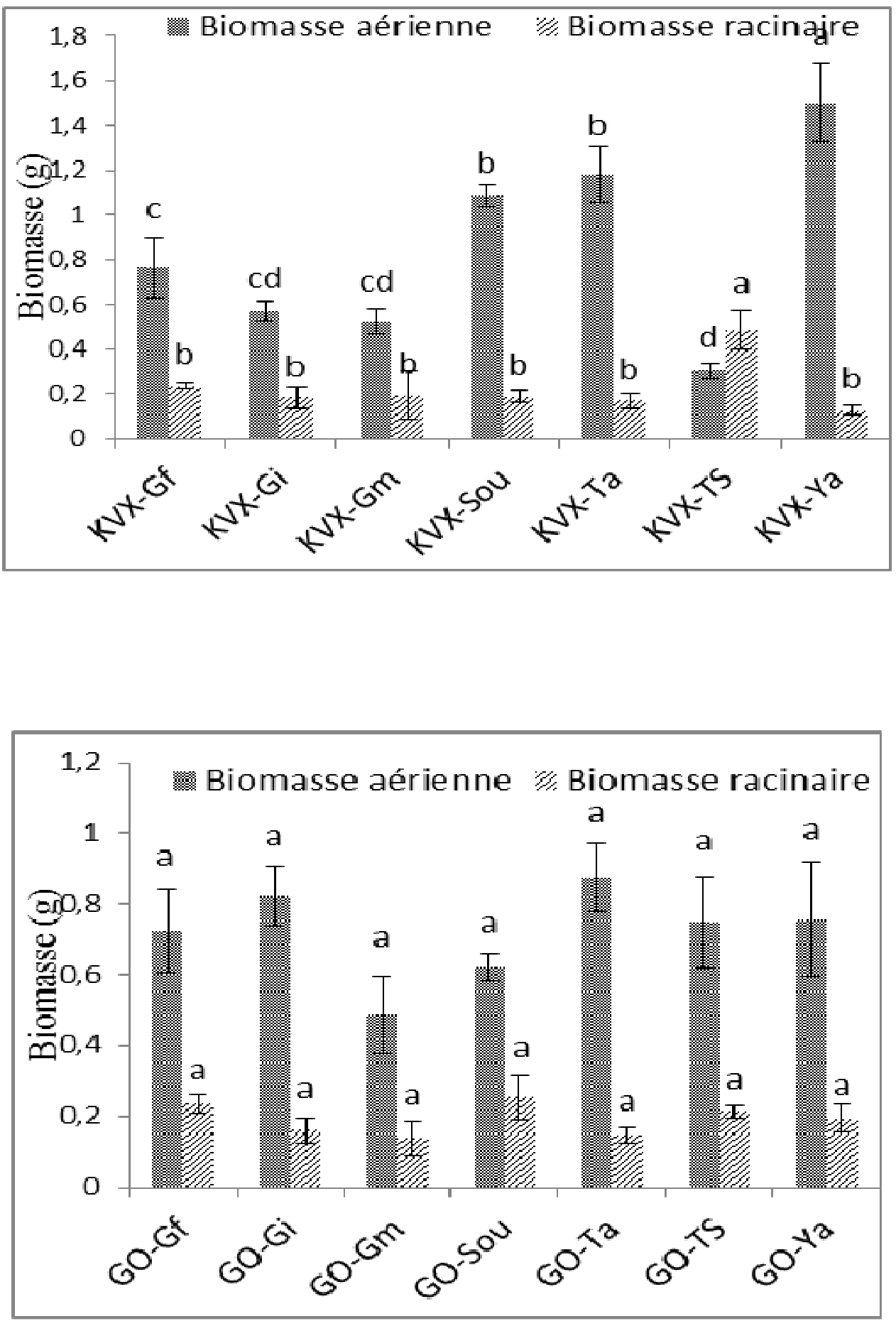

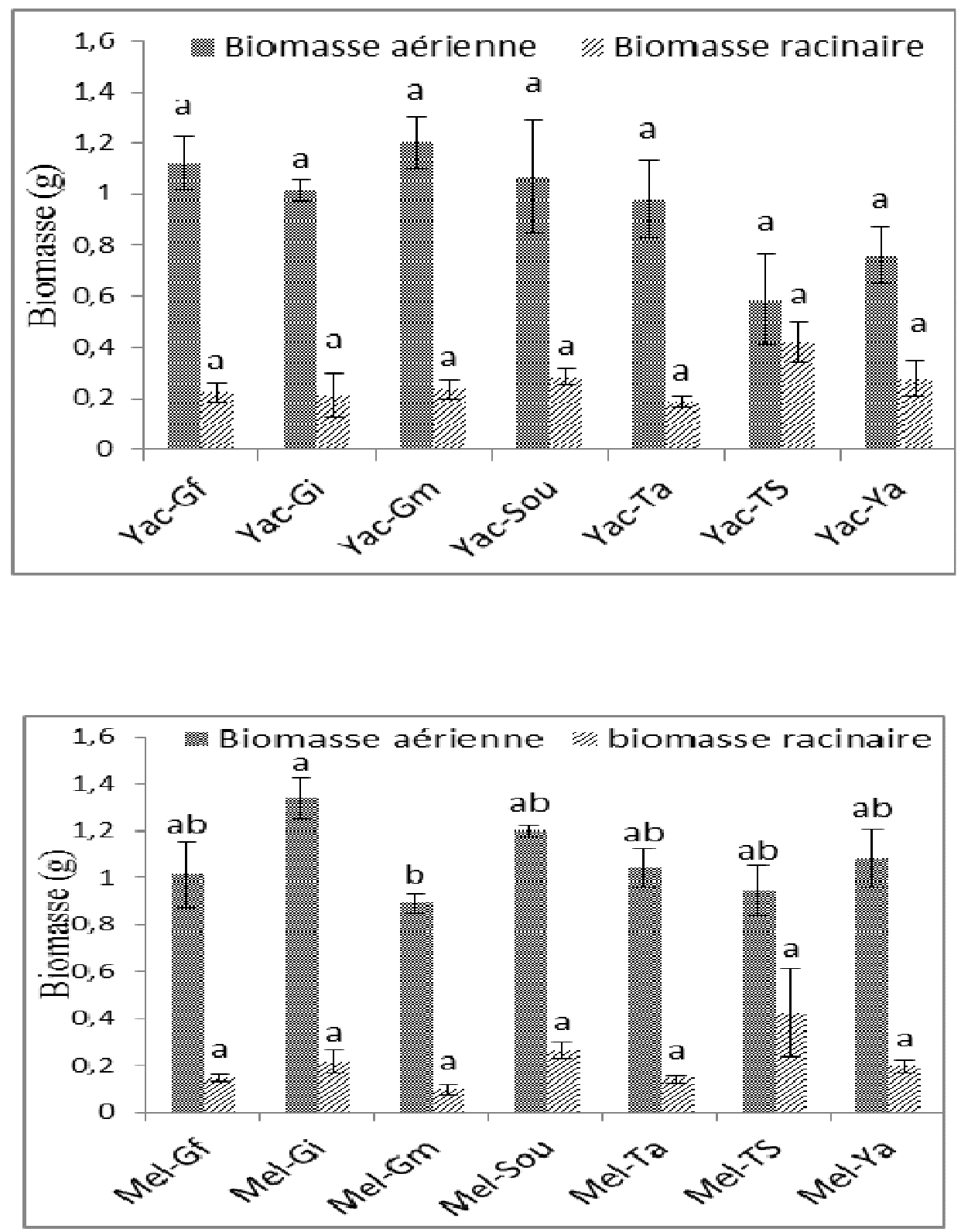

Figure 3: Biomasses aérienne et racinaire après 60 jours de semis des variétés de niébé (Gorom local : GO, KVX396-4-5-2D : KVX; Mélakh: Mel et Yacine: Yac) inoculées ou non avec les souches de CMA. (Glomus mossea : Gm, Glomus fasciculatum : Gf, Glomus intraradices : Gi, talé mossi 1: Ta, yakouta 1: Ya et soumousso1 : Sou). TS= témoin stérile. Pour le même paramètre (biomasse aérienne ou racinaire) et pour la même variété, les valeurs qui ont en commun une même lettre ne sont pas différentes significativement selon le test de NewmanKeuls au seuil de $5 \%$. 


\section{DISCUSSION}

Cette étude a montré que les variétés de niébé utilisées répondaient variablement à l'inoculation mycorhizienne en pépinière.

L'inoculation des plantes a stimulé leur croissance en hauteur. Cette stimulation est plus perceptible à la fructification, période de forte demande en éléments minéraux pour les plantes. Etant donné que la symbiose améliore la nutrition hydrique et minérale, la plante ne trouvera pas de nécessité de former cette symbiose si les éléments nutritifs sont disponibles dans le milieu. Cette symbiose s'établira et se développera progressivement avec l'épuisement des nutriments directement accessibles aux racines des plantes dans le sol (Dommergues et Mangenot, 1970 ; Gianinazzi-Pearson et Gianinazzi, 1986 ; Plenchette, 1982 ; Strullu, 1991).

Les résultats sur la biomasse ont montré que les productions en matière sèche sont améliorées par l'inoculation avec ou non des différences significatives par rapport aux témoins. Des réponses analogues ont été signalées dans le cas de Leucaena inoculée avec Glomus sp., du trèfle inoculé avec $G$. mosseae et, dans le cas de Acacia nilotica et Acacia senegal inoculées avec un complexe mycorhizien de souches indigènes (Dixon et al., 1993 ; Laaziza et al., 2003 ; Laminou et al., 2009). La production de biomasse dans certains cas (KVX+Ya à 60 jours ;

Figure) est plus stimulée par l'inoculation avec les souches indigènes qu'avec les souches de référence. Des résultats similaires ont été obtenus avec Acacia nilotica et Acacia senegal (Laminou et al., 2009).

Pour les variétés du Sénégal, les analyses statistiques ne montrent aucune différence significative entre les plants inoculés et ceux non inoculés ni pour la biomasse aérienne et racinaire, ni pour la hauteur. Ces variétés seraient moins sensibles à l'inoculation. En effet, même si les champignons endomycorhiziens ne montrent guère de spécificité d'hôte, les populations mycorhiziennes semblent adaptées à des conditions édaphiques et climatiques données (Lambert et al., 1980) et la variabilité de la réponse de ces variétés de niébé à l'inoculation mycorhizienne pourrait se justifier par une préférence d'hôte des champignons endomycorhiziens (Diop et al., 2003 ; Khalil et al., 1994). Aussi, l'efficacité de l'inoculation ne se traduit pas toujours par une amélioration de la croissance des plantes hôtes (Guissou et al., 2001), mais peut améliorer la nutrition minérale et phosphatée en particulier (Smith et al., 2003). Une analyse de la teneur en éléments minéraux contenus dans la partie aérienne aurait permis d'évaluer l'effet des différentes souches sur la nutrition des variétés testées.

En général, la biomasse racinaire à 60 jours est inférieure à celle observée à 25 jours. Ceci s'explique par le fait que le niébé est une plante annuelle. Ainsi, à la fin de son cycle végétatif, il y a un vieillissement du système racinaire qui pourrait avoir pour conséquence une perte en partie de racine, donc de biomasse racinaire.

Malgré le faible taux de mycorhization des souches indigènes par rapport aux souches de référence, en particulier à la fructification, les résultats relatifs à la croissance en hauteur et à la biomasse totale produite montrent que les apports de certaines souches indigènes (Ya) ont induit plus d'effets que les souches de références qui ont pourtant des taux et intensités de mycorhization plus élevés. La stimulation de la croissance des plantes ne va donc pas de pair avec l'intensité et la fréquence de mycorhization. Cela pourrait s'expliquer par le fait qu'il n'est pas nécessaire que le niveau d'infection soit très élevé pour être bénéfique à la plante, particulièrement dans les régions semi-arides (Diagne et Ingleby, 2003). Moore (1998) affirme qu'audelà de $12 \%$ de colonisation racinaire, les avantages tirés par la plante hôte ne sont plus sensibles. Hetrick et al. (1992) observent 
également que la croissance des plantes n'est pas forcement liée au degré de colonisation de leurs racines par des CMA. Il faut aussi signaler que nous avons dénombré au niveau des inoculums indigènes au moins 2 genres de CMA [Ya (Acaulospora et Glomus), Ta (Gigaspora et Glomus) et Sou (Glomus et Acaulospora)] (Haro, 2011) dont les effets peuvent s'ajouter par rapport aux inoculums monospécifiques.

Nos résultats concordent avec ceux de Greipsson et EL-Mayas (2000) qui ont observé que la croissance des plants de Leymus arenarius est significativement améliorée par un inoculum de CMA indigène comparé à des inoculums commerciaux monospécifiques. Aussi, au Sénégal, l'utilisation d'isolats indigènes de CMA a permis d'améliorer significativement la surface foliaire et la biomasse racinaire des plants de différentes variétés de Sésame d'au moins $80 \%$ (Diouf et al., 2009).

Cette étude a montré que l'utilisation des souches mixtes indigènes conviendrait mieux que des souches de CMA monospécifiques de référence, en particulier pour les deux variétés du Burkina Faso. L'inoculation n'est bénéfique que si les souches utilisées sont plus compétitives que les souches existantes dans le sol (Bâ et al., 1996). D'après Coperman et al. (1996), le comportement des champignons mycorhiziens et leur efficacité seraient liés à leur milieu d'origine et précisément aux facteurs environnementaux de leurs habitats. Il apparaît donc que la mycorhization par les souches indigènes doit être privilégiée, car mieux adaptées aux conditions environnementales. Par contre, pour les variétés sénégalaises, en absence de données sur la nutrition minérale des plants inoculés, aucun des inoculums utilisés ne peut être sélectionné à ce stade.

En plus de la symbiose mycorhizienne, le Niébé vit également en symbiose avec des bactéries fixatrices d'azote atmosphérique
(Rhizobiums). Les travaux de Megueni et al. (2011) ont mis en évidence l'importance de la double inoculation rhizobium-CMA dans l'amélioration de la production foliaire de deux variétés de Niébé ainsi que la teneur en protéine, $\mathrm{Ca}, \mathrm{K}$ et $\mathrm{Fe}$. Il serait donc intéressant de tenir compte de ces deux symbioses pour l'amélioration de la productivité du Niébé au champ.

\section{REMERCIEMENTS}

Ce travail a bénéficié du soutien du projet CORUS-6121 MYCOVIGNA financé par le Ministère Français des Affaires Etrangère et Européenne (MAEE).

\section{REFERENCES}

Bâ AM, Dalpé Y, Guissou T. 1996. Les Glomales d'Acacia holosericea et d'Acacia mangium. Bois et Forêts des Tropiques, 250: 5-18.

Bado BV. 2002. Rôle des légumineuses sur la fertilité des sols ferrugineux tropicaux des zones guinéenne et soudanienne du Burkina Faso. Thèse de PhD, Université Laval, Québec, Canada, p. 167.

Coperman RH, Martin CA, Stutz JC. 1996. Tomato growth in response to salinity and mycorrhizal fungi from saline or nonsaline soils. Hortic. Sci., 31: 341-344.

Diagne O, Ingleby K. 2003. Ecologie des champignons mycorhiziens arbusculaires infectant Acacia raddiana. In Un Arbre au Désert. IRD (ed): Paris; 205-228.

Diop TA, Wade TK, Diallo A, Diouf M, Gueye M. 2003. Solanum Cultivar Responses to Arbuscular Mycorrhizal Fungi: Growth and Mineral Status. African Journal of Biotechnologies, 2: 429-443.

Diouf M, Boureima S, Diop TA. 2009. Réponse de deux variétés de sésame à l'inoculation avec des champignons mycorhiziens arbusculaires candidats. Agronomie Africaine 21(1): 37-47.

Dixon RK, Rao MV, Garg VK. 1993. Inoculation of Leucaena and Prosopis seedlings with Glomus and Rhizobium 
species in saline soil: Rhizosphere relations and seedling growth. Arid Soil, Res. Rehabil., 7: 133-144.

Dommergues YR, Mangenot F. 1970. Ecologie Microbienne du Sol. Edition Masson: Paris; 796 p.

Egli S, Brunner I. 2002. Les mycorhizes: une fascinante biocénose en forêt. WSL Birmensdorf, 1-8.

Gianinazzi-Pearson V, Gianinazzi S. 1986. The physiology of improved phosphate nutrition in mycorrhizal plants. In Les Mycorhizes, Physiologie et Génétique. INRA: Paris; 101-109.

Greipsson S, El-Mayas H. 2000. Arbuscular mycorrhizae of Leymus arenarius on coastal sands and reclamation sites in Iceland and response to inoculation. Restoration Ecol., 8: 144-150.

Guissou T, Bâ AM, Plenchette C, Guinko S, Duponnois R. 2001. Effets des mycorhizes à arbuscules sur la tolérance a un stress hydrique de quatre arbres fruitiers : Balanites aegyptiaca (L) Del., Parkia biglobosa (Jacq.) Benth., Tamarindus indica L. et Zizyphus mauritiana Lam. Sécheresse, 2(12): 121127.

Haro H. 2011. Effet d'inoculums de champignons mycorhiziens arbusculaires sur la productivité du niébé Vigna unguiculata (L.) Walp. Mémoire de DEA, Université de Ouagadougou, p. 80.

Hetrick BDA, Wilson GWT, Cox TS. 1992. Mycorrhizal dependency of modern wheat varieties, landraces and ancestors. Can. J. Bot., 70: 2032-2040.

Hewitt EJ. 1966. Sand and Water Culture Methods Used in the Study of Plant Nutrition $\left(2^{\text {nd }}\right.$ edn). East Malling, Eng, Commonwealth Bureau of Horticulture and plantation crops, Tech. Comm 22. Rev.

Khalil S, Loynachan TE, Tabatabai MA. 1994. Mycorrhizal Dependency and Nutrient Uptake by Improved and Unimproved Corn and Soybean Cultivars, Agronomy Journal, 86: 949 - 958.
Laaziza BK, Gomez AM, Quarraqi El M, Oihabi A. 2003. Réponses physiologiques et biochimiques du trèfle (Trifolium alexandinum. L.) à la double association mycorhizes rhizobiums sous une contrainte saline. Agronomie, 23: 571580.

Lambert DH, Cole HJr, Baker DE. 1980. Adaptation of vesicular-arbuscular mycorrhizae to edaphic factors. New Phytol., 85: 513-520.

Laminou MO, Ibrahim D, Campanella B, Paul R. 2009. Effets de l'inoculation mycorhizienne et du substrat sur la croissance et la résistance au stress hydrique de cinq espèces fixatrices de dunes : Acacia raddiana Savi ; Acacia nilotica (L.) Willd. Ex Del. var. adansonii ; Acacia senegal (L.) Willd; Prosopis chilensis Stunz. et Bauhinia rufescens Lam.. Geo. Eco. Trop., 33: 115124.

Marx DH. 1969. The influence of ectotrophic mycorrhizal fungi on the resistance of pine roots to pathogenic infections. I. Antagonism of mycorrhizal fungi to root pathogenic fungi and soil bacteria. Phytopathol., 59: 153-163.

Megueni C, Awono ET, Ndjouenkeu R. 2011. Effet simultané de la dilution et de la combinaison du Rhizobium et des mycorhizes sur la production foliaire et les propriétés physico-chimiques des jeunes feuilles de Vigna unguiculata (L.) Walp. J. Appl. Biosci., 40: 2668 - 2676.

Moore JC. 1998. Plant succession in semi-arid grasslands and response to mycorrhizal colonization. Abstracts of Ecological Society of America Meeting, Davis, CA, USA, p. 312.

Mosse B. 1973. Advances in the study of Vesicular-Arbuscular Mycorrhiza. Annu. Rev. Phytopathol., 11: 171 -196.

Ogbuinya PO. 1997. Advances in Cowpea Research. Biotech. Dev. Monitor, 33: 1012.

Ouédraogo JT, Drabo I, Tignegre JB, Dabire C, Sereme P, Konate G. 2003. Fiches 
techniques du niébé, variétés kvx 396-45-20, kvx 61-1, kvx745-11P, Gorom local. INERA, Burkina Faso.

Phillips JM, Hayman DS. 1970. Improved procedures for clearing roots and staining parasitic and vesicular-arbuscular mycorrhizal fungi for rapid assessment of infection. Trans. Br. Mycol. Soc., 5: 158161.

Plenchette C. 1982. Recherches sur les endomycorhizes à vésicules et arbuscules. Influence de la plante hôte, du champignon et du phosphore sur l'expression de la symbiose endomycorhizienne. Thèse de $\mathrm{PhD}$, Université Laval, Québec, Canada, p. 182.

Plenchette C, Clermont-Dauphin C, Meynard JM, Fortin JA. 2005. Managing arbuscular mycorrhizal fungi in cropping systems. Can. J. Plant Sci., 85: 31-40.

Rahman, MK, Parsons JW. 1997. Effects of inoculation with Glomus mosseae, Azorhizobium caulinodans and rock phosphate on the growth of and nitrogen and phosphorus accumulation in Sesbania rostrata. Biol. Fertil. Soils, 25: 47-52.

Rahman MK, Kabir SM, Mohsini GM, Didarul AM. 2006. Interaction of arbuscular mycorrhizal fungus Glomus mossae and phosphorus on growth and nutrient uptake of maize plants grown under different soil conditions. Bangladesh J. Bot., 35(1): 1-7.

Singh BB, Chambliss OL, Sharma B. 1997. Recent advances in cowpea breeding. In Advances in Cowpea Research, Singh BB, Mohan Raj DR, Dashiell KE, Jackai LEN (eds). Co-publication of International Institute of Tropical Agriculture (IITA) and Japan International Research Center for Agricultural Sciences (JIRCAS): Ibadan, Nigeria; 30-49.

Smith SE, Smith FA, Jakobsen I. 2003. Mycorhizal fungi can dominate supply to plants irrespective of growth response. Plant Physiol., 133: 16-20.

Smith S, Read D. 1997. Mycorrhizal Symbiosis, (2nd edn). San Diego, CA, Academic Press: New York ; p. 605.

Strullu DG. 1991. Les Mycorhizes des Arbres et des Plantes Cultivées. Edition Lavoisier: Paris, France; p. 250.

Trouvelot A, Kough JL, Gianinazzi-Pearson V. 1986. Mesure du taux de mycorhization VA d'un système radiculaire. In Aspects Physiologiques et Génétiques des Mycorhizes. Edition INRA: Dijon, France; 217 - 221. 